\title{
Comparison of narrow-band UVB vs. psoralen with UVA phototherapy for early-stage mycosis fungoides
}

\author{
Amrita Goyal \\ Department of Dermatology, University of Minnesota, Minneapolis, MN, USA \\ Correspondence to: Amrita Goyal, MD. University of Minnesota, 516 Delaware St SE, Minneapolis, MN 55455, USA. Email: Goyal046@umn.edu. \\ Provenance: This is an invited article commissioned by the Academic Editor Dr. Weijun Jiang (Nanjing Normal University, Department of \\ Reproductive and Genetics, Institute of Laboratory Medicine, Jinling Hospital, Nanjing University School of Medicine, Nanjing, China). \\ Comment on: Phan K, Ramachandran V, Fassihi H, et al. Comparison of Narrowband UV-B With Psoralen-UV-A Phototherapy for Patients With \\ Early-Stage Mycosis Fungoides: A Systematic Review and Meta-analysis. JAMA Dermatol 2019;155:335-41.
}

Submitted Nov 18, 2019. Accepted for publication Nov 29, 2019.

doi: $10.21037 / \mathrm{atm} .2019 .12 .09$

View this article at: http://dx.doi.org/10.21037/atm.2019.12.09

Mycosis fungoides (MF) is a rare cutaneous T-cell lymphoma that typically presents in its early stages with papulosquamous patches and plaques in a sun-protected distribution on the skin. MF may progress to late-stage disease including cutaneous tumors as well as blood, lymph node, or visceral involvement.

The first-line management for early stage MF (stage IA-IIA) includes either skin-directed or systemic therapies such as topical corticosteroids, topical chemotherapeutic agents, oral or topical retinoids, and oral methotrexate. Additionally, non-pharmacologic modalities include phototherapy: narrow-band UVB (nbUVB) or UVA with psoralen (PUVA). Late-stage disease (IIB-IVB) requires treatment escalation to systemic chemotherapy, localized ionizing radiotherapy, total skin electron beam radiotherapy, extracorporeal photophoresis, or allogeneic hematopoietic stem cell transplant.

The protocol for nbUVB involves the localized exposure of affected areas to 311 nanometers $(\mathrm{nm})$ photons with treatments administered 2-3 times weekly for several months. nbUVB is thought to interfere with immunity via alteration of Langerhans cell behavior $(1,2)$. Exposure to this wavelength results in decreased antigen presentation and upregulation of interleukin (IL)-2, IL-6, and TNF. T-cell apoptosis may also be implicated in suppression of neoplastic activity (3).

PUVA requires the use of oral or topical psoralen to sensitize cells to UVA-band photons (320-340 nm) by intercalating into the DNA of normal and tumor cells.
Psoralen has improved efficacy on tumor cells due to an increased rate of uptake and incorporation related to a higher mitotic rate $(1,4,5)$. On exposure to UVA, the psoralen induces the formation of cross-links between DNA strands. The psoralen can also directly generate oxygen free-radicals, resulting in DNA or cellular damage $(1,4,5)$. Given these mechanisms, PUVA is thought to "penetrate deeper" into the skin and be more suitable for thick plaques or folliculotropic disease.

To date, there have been few studies available to compare the efficacy and toxicity of the two treatments. To fill this knowledge gap, Phan et al. performed a systematic review and meta-analysis to investigate the comparative safety and efficacy between PUVA and nbUVB in patients with earlystage MF (6).

Eligible studies directly compared cohorts of patients who underwent either nbUVB to PUVA as treatment of early stage MF. Excluded were studies were those profiling the treatment of patients with advanced-stage disease (stage IIB-IVB), pediatric age, or noncomparative studies. This meta-analysis identified seven studies, most of which were observational in nature, and were assessed to be poor to moderate quality. In total, these studies included a total of 778 patients [ 405 of $724(55.9 \%)$ men, mean age 52 years], 251 of whom underwent nbUVB with the remainder, 527 patients, treated with PUVA.

Response to therapy was defined as complete or partial response. For cases treated with PUVA, 479/527 (90.9\%) demonstrated some amount of response, whereas 
$220 / 251(87.6 \%)$ treated with nbUVB had some amount of treatment response $(\mathrm{P}=0.2)$. There was no significant difference in treatment effect when stratified for stage.

It was found that $73.8 \%(389 / 527)$ of patients treated with PUVA had a complete response, compared with $62.2 \%$ (156/251) patients treated with nbUVB $(\mathrm{P}=0.04)$. Partial response rate was similar for the two groups, $18.0 \%(90 / 501)$ for PUVA and $28.7 \%$ (64/223) for NBUVB ( $\mathrm{P}=0.07)$. Failed response was similar between the two groups, $8.3 \%$ (44/527) for PUVA and $11.2 \%$ (28/251) for nbUVB ( $\mathrm{P}=0.36)$. Finally, relapse free survival for PUVA was longer than for nbUVB, with median 33.4 months for PUVA as compared to 14.9 months for nbUVB.

Overall, this meta-analysis demonstrates that PUVA and nbUVB demonstrate similar efficacy when all response categories are considered. PUVA had a higher rate of complete response than nbUVB reaching statistical significance with a corresponding point estimate of $11.6 \%$. PUVA also showed an increased period of disease freedom in the two studies available for analysis corresponding to a hazard ratio of 1.93 (95\% CI, 1.07-3.49). The toxicity assessment showed no significant differences in acute adverse effects (erythema, nausea, pruritus, phototoxic effects, dyspepsia, pain). Notably, they did not consider the long-term side-effects of the development of melanoma and non-melanoma skin cancers.

In clinical practice, there are several limitations to the use of PUVA, many of which are related to the administration of psoralen itself. Patients who undergo psoralen treatments must fastidiously protect their body surface (including eyes) from the sun for the remainder of the treatment day. When taken orally, psoralen can cause nausea, vomiting, headache, phototoxicity, and immunosuppression. It must also be taken at a precise time before phototherapy, which can pose logistical difficulties for patients. Availability of PUVA treatment facilities may be limited. Finally, repeated PUVA exposure (typically $>200$ treatments) carries with it the risk of development of melanoma and non-melanoma skin cancers, a risk not believed to be associated with nbUVB.

In comparison to PUVA, nbUVB is much more widely available, however it too has its own acute toxicity profile including erythema, blistering, pruritus, xerosis, and reactivation of herpes simplex virus 1 and 2 . The difference in toxicity profile is largely related to the omission of psoralen from the treatment protocol, obviating the aforementioned inconveniences of this component of treatment. The best available data do not support any increased risk of skin cancer for nbUVB treatments.
One of the notable limitations of this study is that it does not specifically consider patients with the categories of disease for which PUVA is generally felt to be the most strongly indicated: patients specifically with thicker plaques and those with folliculotropic disease. An assessment of the efficacy of nbUVB versus in these populations would be of particular utility. Other limitations include the fact that this is a meta-analysis, and limited by the strength of the papers on which it is based which were, again, of poor to moderate quality by the author's assessment.

In summary, this paper examines the relative efficacy and side effects of nbUVB and PUVA for early-stage MF. It concludes that they are roughly equivalent, with a slightly higher complete response rate for PUVA. It provides the best available evidence to date given the lack randomizedcontrolled trials to answer this important clinical question.

\section{Acknowledgments}

The author would like to thank Dr. Krishnan Patel of the Department of Radiation Oncology at the University of Minnesota for his editorial contributions.

\section{Footnote}

Conflicts of Interest: The author has no conflicts of interest to declare.

Ethical Statement: The author is accountable for all aspects of the work in ensuring that questions related to the accuracy or integrity of any part of the work are appropriately investigated and resolved.

\section{References}

1. Duthie MS, Kimber I, Norval M. The effects of ultraviolet radiation on the human immune system. Br J Dermatol 1999;140:995-1009.

2. el-Ghorr AA, Norval M. Biological effects of narrow-band (311 nm TL01) UVB irradiation: a review. J Photochem Photobiol B 1997;38:99-106.

3. Krutmann J, Morita A. Mechanisms of ultraviolet (UV) B and UVA phototherapy. J Investig Dermatol Symp Proc 1999;4:70-2.

4. Okamoto H, Takigawa M, Horio T. Alteration of lymphocyte functions by 8 -methoxypsoralen and longwave ultraviolet radiation. I. Suppressive effects of PUVA on T-lymphocyte migration in vitro. J Invest Dermatol 
1985;84:203-5.

5. Pathak MA, Joshi PC. The nature and molecular basis of cutaneous photosensitivity reactions to psoralens and coal tar. J Invest Dermatol 1983;80:66s-74s.

6. Phan K, Ramachandran V, Fassihi H, et al. Comparison of

Cite this article as: Goyal A. Comparison of narrow-band UVB vs. psoralen with UVA phototherapy for early-stage mycosis fungoides. Ann Transl Med 2019;7(Suppl 8):S268. doi: 10.21037/atm.2019.12.09
Narrowband UV-B With Psoralen-UV-A Phototherapy for Patients With Early-Stage Mycosis Fungoides: A Systematic Review and Meta-analysis. JAMA Dermatol 2019;155:335-41. 